\title{
Making a frothy shampoo or beer
}

\author{
Douglas J. Durian and Srinivasa R. Raghavan
}

quick. study

\author{
Both beer and shampoo have polar molecules that accumulate at a \\ liquid-gas interface, but the mechanisms that prevent bubble collapse \\ in soap foams or pilsner heads are fundamentally different.
}

Doug Durian is a professor of physics and astronomy at the University of Pennsylvania in Philadelphia. Srini Raghavan is an associate professor of chemical and biomolecular engineering at the University of Maryland, College Park.

Why do certain liquids tend to foam while others do not? For example, when you vigorously shake pure water you'll produce bubbles, but they rapidly coalesce and disappear. Shampoo or beer, on the other hand, can have foam that persists for several minutes or even hours. What ingredients in shampoo and beer make their foams stable, and what physical concepts control that stability?

\section{Foams evolve}

Rub shampoo into wet hair and you'll form a lather containing small air bubbles. You can achieve the same effect by simply shaking a bottle of dilute shampoo or detergent solution. The act of pouring will generate foam in beer, but even once the beer is poured, dissolved gas, chiefly carbon dioxide, will break out into small bubbles at nucleation sites along the glass wall. Beer bubbles and other bubbles that form in a pool

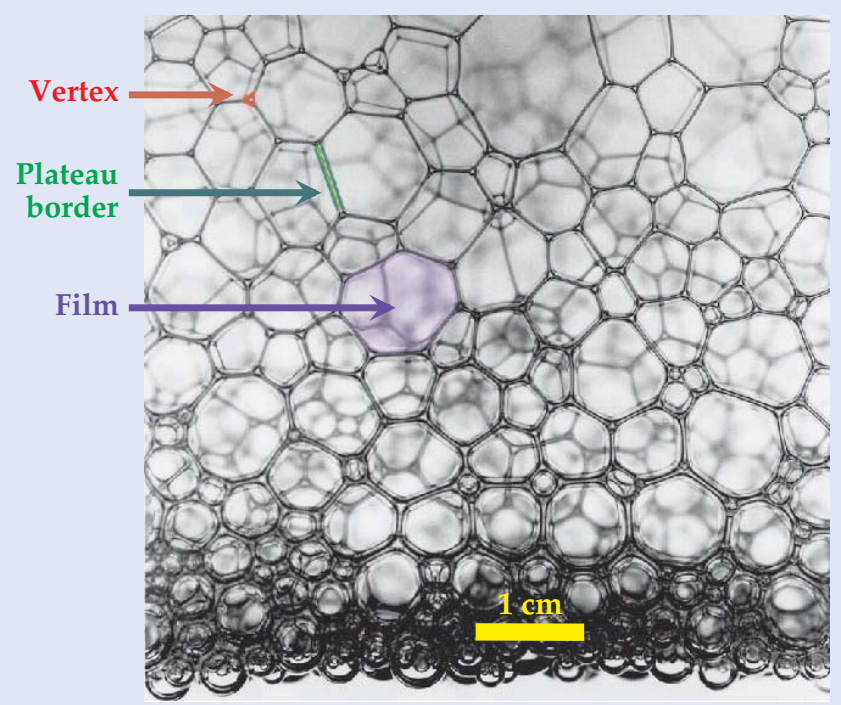

Figure 1. Foam structures. This photograph, taken 30 minutes after a detergent solution was shaken, shows several elements of foam geometry. The films toward the top, where the foam is dry, intersect at so-called Plateau borders that in turn intersect at vertices. Dry foams are made of polyhedral bubbles; wet foams, as seen near the bottom, have rounder bubbles because liquid has inflated the Plateau borders and vertices. of liquid rise and collect as a layer of foam on top of the liquid (see the article by Neil Shafer and Richard Zare, PHYSICS TODAY, October 1991, page 48).

If bubbles do not immediately coalesce, the liquid can drain down and produce a structure like that shown in figure 1. Such gravitational drainage is destabilizing; it separates the gas and liquid phases and brings bubbles close enough to merge. The process may be slowed by increasing the liquid viscosity or by decreasing the bubble size, but it cannot be stopped completely, at least not on Earth. A second destabilizing mechanism is Ostwald ripening, also known as coarsening or disproportionation, whereby gas diffuses from smaller to larger bubbles. It occurs because, as described by Laplace's law, the gas is at a higher pressure in the more highly curved, smaller bubbles. Ostwald ripening cannot be stopped either; however, it can be slowed if the gas solubility or diffusivity is decreased. Nitrogen, for example, is less soluble in water than $\mathrm{CO}_{2}$. By introducing just 20 parts per million of $\mathrm{N}_{2}$ in beer, a brewer can substantially enhance foam stability - a trick often used for English and Irish ales. The resulting smaller bubbles also yield a head whose texture is rich and creamy.

The random structures formed by packed bubbles, as seen in figure 1, are largely independent of bubble size and chemical composition. Such beautiful geometry has inspired several recent lines of research. Those include uncovering how bubble growth rates vary with the number of bubble sides and minimizing the surface area per unit volume. Other investigators are exploring bubble jamming - the formation of solidlike states in which bubbles don't change neighbors over experimentally long times. Bubble jamming exhibits universal features that may be compared with those in systems of sand grains, colloidal particles, and glassy molecular liquids.

\section{Charge separation stabilizes shampoo foam}

The stability of any foam ultimately relies on the hindrance of bubble coalescence. Gravitational drainage, evaporation, and van der Waals forces can all reduce the thickness of the liquid films between bubbles to the point of bursting. In shampoos and detergents, stabilization arises from surfactant molecules that coat the bubbles. As figure 2a shows, surfactants have a split personality, with a water-loving head and an oil-loving tail. To minimize energy, the surfactants shield their tails from water by adsorbing at the air-water interfaces or, when in solution, aggregating into micelles. Sur- 
a

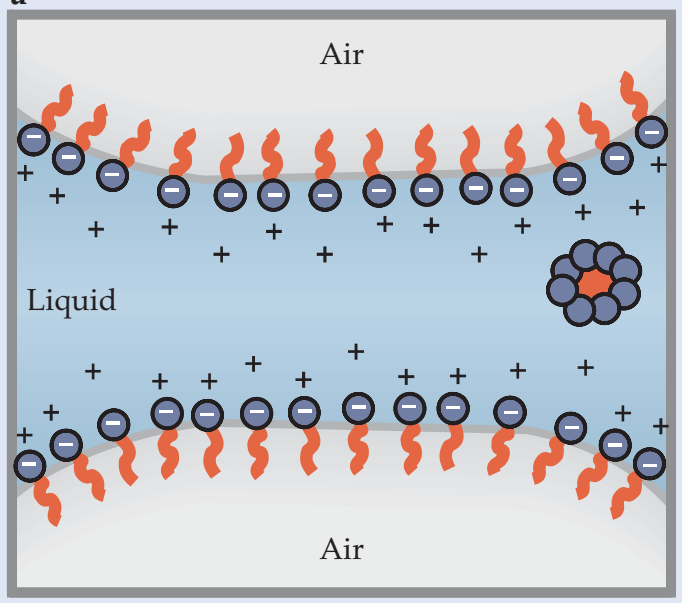

b

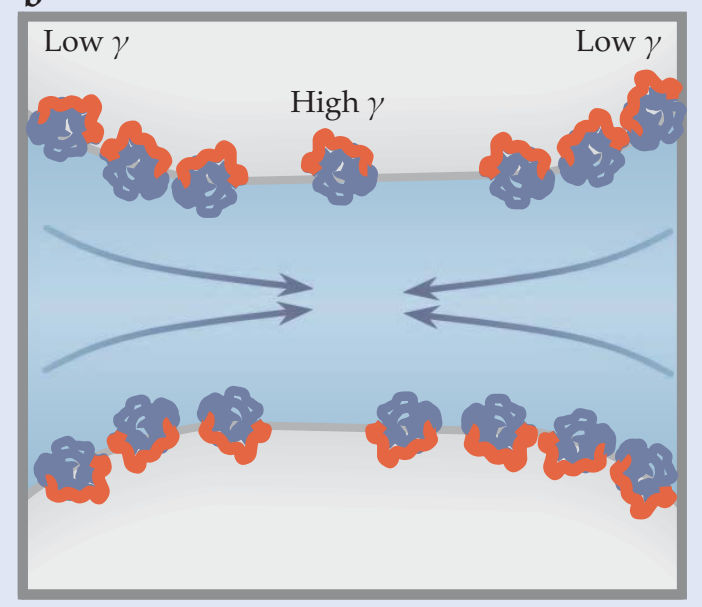

Figure 2. Stability mechanisms. (a) Adsorbed ionic surfactants and their associated electrostatic repulsions stabilize detergent films. The hydrophobic tails of the surfactants (red) are shielded from water, for both the adsorbed surfactants and those aggregated into micelles, where they are surrounded by hydrophilic

heads (blue). (b) Beer films are stabilized by adsorbed proteins and the resulting Gibbs-Marangoni effect, which causes the flow from low to high surface tension $(\gamma)$ indicated by the arrows. As with surfactants, proteins have hydrophilic (blue) and hydrophobic (red) parts. factant adsorption significantly reduces the energy per unit area, or surface tension $\gamma$, of the air-water interface-from about $80 \mathrm{erg} / \mathrm{cm}^{2}$ for a clean interface to about $30 \mathrm{erg} / \mathrm{cm}^{2}$ for one that is fully coated.

Surfactants with ionizable heads are key to creating a repulsive force that counteracts the van der Waals attraction and thus stabilizes a soap film. When a head dissociates, one charge remains stuck at the interface, and the other, attracted to the surface by electrostatic forces, wanders only a short distance away. The result is a double-layer distribution of charges - for example, a plane of negative heads and an adjacent diffuse cloud of positive counterions. A film has two such double layers. If the film gets too thin, the diffuse charge clouds overlap and repel, just as two neutral atoms do when their electron clouds interact. The opposing electrostatic and van der Waals forces are typically balanced for film thicknesses in the range of $10-1000 \mathrm{~nm}$. The wavelengths of visible light lie squarely in that range; the familiar result is that isolated soap bubbles display shimmering interference colors. In bulk foam, soap films adjust to equilibrium thickness by shunting excess liquid into Plateau borders and vertices.

Shampoo foam stability is thus promoted by an electrostatic double-layer repulsion of opposite sides of the soap film between neighboring bubbles. For maximum effectiveness, the surfactant heads should be highly ionized. Also important is that the surfactant concentration be greater than the so-called critical micelle concentration, so that the interfaces are fully coated and the solution has plenty of micelles to provide surfactant for quickly coating newly created bubbles.

\section{Tension gradients stabilize beer foam}

No one wants soap in beer, but everyone wants the suds. Fortunately, beer contains surface-active proteins left over from the malted barley and wheat used in the brewing process. Those globular proteins, most importantly a relatively small one called lipid transfer protein 1 and a larger one called Z23, are less ionizable and less plentiful than the surfactants in shampoo. Therefore, electrostatic repulsion is generally not strong enough to support stable equilibrium beer films. Instead, a dynamical mechanism known as the GibbsMarangoni effect comes into play. As illustrated in figure $2 b$, a suddenly stretched film has a lower protein concentration and hence a higher local surface tension. The gradient in surface tension induces a flow of liquid that helps to heal the thin spot and hinder rupture.

Beer foam stability is thus promoted by a strong GibbsMarangoni effect, which is optimized when absorbed proteins are efficient at changing the surface tension. It is also important to prevent fresh regions of interface from being replenished by migration of dissolved proteins from the bulk liquid, which would short-circuit the healing effect of the Gibbs-Marangoni flow. To achieve optimal stability, the solution $\mathrm{pH}$ should be close, but not too close, to the "isoelectric point" at which the proteins are uncharged. If the solution is too far from the IEP, the proteins are highly soluble and surface tension gradients never develop; too close, and the proteins precipitate out. Wheat beers like Belgian wit and German hefeweizen are often cloudy because of light scattered by precipitated proteins.

Unlike detergent foams, which famously stand up to grime, beer foams can be destabilized with a bit of oil. That relative fragility arises both because the electrostatic repulsion is small and because the Gibbs-Marangoni mechanism is easily disrupted. It is well known to enthusiasts that a glass must be "beer clean" - free of any grease or skin oils, including fingerprints - to support a good head of foam. Even contact with lipstick may be enough to collapse the head. The fascinating physics of foams offers much to contemplate as you shower before heading off to the bar.

\section{Additional resources}

J. H. Aubert, A. M. Kraynik, P. B. Rand, "Aqueous Foams," Sci. Am., May 1986, p. 74.

- D. Weaire, S. Hutzler, The Physics of Foams, Clarendon Press, New York (1999).

- R. D. MacPherson, D. J. Srolovitz, "The von Neumann Relation Generalized to Coarsening of Three-Dimensional Microstructures," Nature 446, 1053 (2007).

- A. J. Liu, S. R. Nagel, eds., Jamming and Rheology: Constrained Dynamics on Microscopic and Macroscopic Scales, Taylor \& Francis, New York (2001).

- J. N. Israelachvili, Intermolecular and Surface Forces, 2nd ed., Academic Press, New York (1992). 